\title{
Descentralização em política de ciência e tecnologia
}

\author{
ALBERTO CARVALHO DA SILVA
}

$\mathrm{C}$

ADA VEZ MAIS ciência e tecnologia são componentes básicos do planejamento nacional em busca de desenvolvimento econômico, diminuição das desigualdades sociais e preservação do meio ambiente. As metas do desenvolvimento científico não mais se limitam à acumulação acadêmica de conhecimento sobre as leis da natureza ou à busca de soluções para problemas específicos; elas se caracterizam como capacidade de formar e usar o conhecimento como nova forma de capital para que cada nação possa manter a sua autonomia e sua competitividade no equilíbrio entre seus pares. As soluções para os problemas de emprego, educação, habitação, saúde, saneamento, crescimento demográfico, migrações estão, em grande parte, vinculadas a inovações em produtos e serviços, por sua vez dependentes de pesquisa.

Acresce que a moderna sociedade do conhecimento é cada vez mais dinâmica mudando com grande rapidez as suas linhas de desenvolvimento, baseadas em uma atividade científica que produz cinco mil novas publicações por dia, gerando um conhecimento que se renova a cada cinco ou seis anos e está disponível de imediato através dos novos meios de comunicação. O número de trabalhadores na área científica vem aumentando com tal rapidez que estão atualmente em atividade $90 \%$ de todos os que até hoje, se dedicaram à ciência. A formação e atualização de um sistema nacional de ciência e tecnologia deixou de ser o esforço episódico de há 50 anos para se converter em uma necessidade contínua e crescente em que a produção, transferência e utilização do conhecimento formam o carro chefe do desenvolvimento econômico e social.

Tomando como exemplo o problema do emprego, as características de sua oferta sofreram grandes mudanças em comparação com a anterior sociedade industrial. Entre tais mudanças cita-se o trabalho à distância, que oferece atualmente, na Alemanha, um milhão de empregos, com consideráveis ganhos de produtividade e competitividade e com tendência a rápido aumento. Nos países desenvolvidos a força de trabalho no setor primário baixou de 50\% para 3\% ao longo do século XX. Ainda na Alemanha, calculase que os setores de agricultura e de manufatura que até há pouco davam 
conta de $48 \%$ e $30 \%$ do emprego, devem baixar para $3 \%$ e $20 \%$ em 2010 enquanto o setor de serviços deve aumentar de $10 \%$ para $20 \%$ e o de informação, de $10 \%$ para $55 \%$.

Essas mudanças implicam modificações substanciais da força de trabalho, com níveis cada vez mais altos de educação e treinamento, um desafio que, nos países em desenvolvimento, é agravado pelo crescimento demográfico. Com o aumento anual de aproximadamente 86 milhões, a população do mundo deverá ultrapassar os atuais 6 bilhões para alcançar 10 bilhões em 2050. Esse aumento, concentrado na Ásia, na África e na AméricaLatina, é acompanhado de migração crescente para o setor urbano agravando ainda mais a demanda por emprego, sem se falar de habitação, saneamento, transporte, consumo de água e energia, proteção do ambiente, serviços de saúde e educação.

Os países desenvolvidos tendem cada vez mais a descentralizar a sua capacidade em produzir conhecimento e recursos humanos, criando condições para que todas as suas regiões se beneficiem.

A França, a partir dos anos 80, vem descentralizando e seu sistema de ciência, tecnologia e inovação que, atualmente, pode ser descrito como um processo de delegação às instituições de pesquisa, às agências encarregadas dos grandes programas, às diversas regiões e aos programas da OECD.

Inglaterra e Estados Unidos, embora disponham de estruturas com o encargo de planejar, financiar, avaliar e acompanhar as atividades em P\&D em nível nacional, já atingiram tal grau de desenvolvimento em suas várias regiões que o problema de centralizar ou descentralizar está superado.

$\mathrm{Na}$ Alemanha, de um investimento global da ordem de 80 bilhões de marcos em ciência e tecnologia, o setor público participa com cerca de 34 bilhões - 19 bilhões pelo governo federal e 15 bilhões pelos 16 estados. Além disso, cerca de 350 universidades e escolas superiores (Hochshule), cinco organizações (1) mantêm nos estados 140 institutos de pesquisa, acrescidos de quase 300 divisões, grupos de trabalho e núcleos.

A par de garantir uma capacidade própria de pesquisa, inovação e competitividade em cada região, tal infra-estrutura atrai uma rede de subsidiárias de multinacionais que investem anualmente em P\&D cerca de 7 bilhões de marcos e criam ao redor de 35 mil empregos (Bundsbericht Forschung, 1996).

Contrastando com essa situação, no Brasil mais de dois terços do sistema de ciência e tecnologia estão concentrados na região Sudeste (tabela 1). 
Tabela 1 - Alguns indicadores da concentração da capacidade de P\&D na região Sudeste

\begin{tabular}{l|c}
\hline \multicolumn{1}{c|}{ Indicador } & $\begin{array}{c}\% \text { do total } \\
\text { na região Sudeste }\end{array}$ \\
\hline Cursos de pós-graduação, mestrado & 64 \\
Cursos de pós-graduação, doutorado & 80 \\
Docentes de pós-graduação & 71 \\
Bolsas de mestrado - CAPES & 63 \\
Bolsas de doutorado - CAPES & 80 \\
Bolsas de doutorado - CNPq & 86 \\
Recursos do CNPq (fomento) & 63 \\
Recursos do PADCT II & 66 \\
FINEP - número de operações sem retorno & 65 \\
FINEP - investimentos em operações sem retorno & 71 \\
PRONEX - no de projetos aprovados em 1996 e 1997 & 79 \\
Número de grupos de pesquisa & 69 \\
Número de empresas com atividade em P\&D & 80 \\
\hline
\end{tabular}

Tabela 2 - Distribuição regional do total de docentes de ensino superior com grau de doutor e do total de cursos de doutorado (em \%)

\begin{tabular}{l|l|l|l|l}
\hline Região & \multicolumn{2}{|c|}{$\begin{array}{c}\text { Total de docentes } \\
\text { com nível de doutor }\end{array}$} & \multicolumn{2}{c}{$\begin{array}{c}\text { Total de programas } \\
\text { de doutoramento }\end{array}$} \\
\hline & $\begin{array}{l}\text { Escolas } \\
\text { públicas }\end{array}$ & $\begin{array}{l}\text { Escolas } \\
\text { particulares }\end{array}$ & $\begin{array}{l}\text { Escolas } \\
\text { públicas }\end{array}$ & $\begin{array}{l}\text { Escolas } \\
\text { particulares }\end{array}$ \\
Norte & 1,8 & 0,7 & 1,4 & - \\
Nordeste & 12,7 & 2,2 & 5,9 & 5,6 \\
Sudeste & 66,1 & 74,3 & 80,2 & 77,8 \\
Sul & 14,2 & 20,1 & 9,8 & 16,6 \\
Centro-Oeste & 5,2 & 2,7 & 2,7 & - \\
\hline Total & 100 & 100 & 100 & 100 \\
\hline
\end{tabular}


Essa situação tende a se agravar dada a dificuldade das regiões menos favorecidas em competir pelos recursos federais, geralmente escassos e incertos, e em formar novos cientistas e fixá-los em uma atividade produtiva. Para tanto muito contribui a escassez local de docentes e pesquisadores com formação adequada.

Atualmente as regiões Norte e Centro-Oeste somam apenas 400 dos 24 mil docentes nacionais com grau de doutor e 25 dos 616 programas de pós-graduação em nível de doutorado (tabela 2).

Agravando a falta de condições locais para a formação de pesquisadores, os novos valores treinados em outros centros do país ou do exterior tendem a não se fixar em seus centros de origem por falta de condições que lhes permitam exercer com eficiência e continuidade uma atividade científica produtiva.

Assim, as regiões menos favorecidas ficam privadas de desenvolver pesquisa aplicada ao seu desenvolvimento e, mesmo, dada a escassez de infra-estrutura tecnológica e de recursos humanos qualificados, transferir e incorporar conhecimentos gerados em outros centros. Para reverter essa situação, cabe a uma política nacional de ciência e tecnologia para o desenvolvimento criar condições para que todas as regiões do país possam gerar novos conhecimentos e incorporá-los ao seu sistema produtivo e, dessa forma, contar com uma força de trabalho com as qualificações necessárias para desempenhar esta função. Trata-se de um esforço que deve ser prolongado e persistente para tornar possível criar as condições que permitam enfrentar os diversos desafios à medida que eles surjam, e não apenas improvisar medidas de emergência quando os desafios aparecem.

A arma que o Brasil desenvolveu para apoiar a descentralização é o seu sistema de fundações e fundos de amparo à pesquisa previstos nas Constituições de 1989 em 24 estados e Distrito Federal (2). A primeira, a Fapesp, já constava da Constituição do estado de São Paulo em 1947 e foi instalada em 1960, aprovando os primeiros auxílios em 1962. A ela se seguiram a Fapergs no Rio Grande do Sul em 1964, a Faperj no Rio de Janeiro em 1980 e a Fapemig de Minas Gerais em 1985 (3). Em alguns estados (SE, TO, SP, SC, MG) é deduzida a quota transferida aos municípios; na Fapepe deduz-se a arrecadação de pelo menos nove impostos, além de salário educação, transferências de convênios e operações de crédito (4). Quatorze estados (5) e Distrito Federal estabeleceram que os recursos devem ser transferidos em duodécimos; sete estados (6) e DF limitam as despesas com administração a $5 \%$ do orçamento e um (MA), a $10 \%$; sete estados (7) asseguram à fundação ou ao fundo administração privativa dos recursos (tabela 3 ). 
Tabela 3 - Percentual mínimo da renda vinculado a fundações ou fundos em apoio de ciência e tecnologia, nas Constituições de 1989, em 22 estados e DF ${ }^{1}$

\begin{tabular}{l|l}
\hline Percentual & \multicolumn{1}{|c}{ Estados } \\
\hline 0.3 & $\mathrm{PA}^{2}$ \\
0.5 & $\mathrm{MA}, \mathrm{SE}, \mathrm{TO}$ \\
1.0 & $\mathrm{PE}, \mathrm{PI}, \mathrm{SP}$ \\
1.5 & $\mathrm{MS}^{3}, \mathrm{RS}, \mathrm{BA}$ \\
2.0 & $\mathrm{AL}, \mathrm{CE}, \mathrm{MT}, \mathrm{PR}, \mathrm{SC}, \mathrm{RJ}^{4}$ \\
2.5 & $\mathrm{ES}, \mathrm{PB}$ \\
3.0 & $\mathrm{AM}, \mathrm{GO}, \mathrm{MG}^{5}$ \\
\hline
\end{tabular}

Notas:

1 As constituições de Acre, Rondônia e Rio Grande do Norte não definem percentual.

2 Instituída com $0,4 \%$.

3 0,5\% no primeiro ano, $1 \%$ no segundo, $1.5 \%$ a partir do terceiro.

4 1.5\% em 1990 e $2 \%$ a partir de 1991 . Nos primeiros 5 anos um terço da dotação, deve ser transferido ao Fundo de Apoio ao Desenvolvimento Tecnológico - Fatec.

5 Em 1995 passou para 1\% mas recebendo 0.5\% em 1995, 0.7\% em 1996, 0.8\% em 1997 e $1 \%$ a partir de 1998 .

A natureza institucional e os vínculos previstos nas Constituições estaduais de 1989 apresentam algumas diferenças entre unidades da Federação (quadro 1). 
Quadro l - Modelos institucionais e vínculos de fundações e fundos previstos nas Constituições

\begin{tabular}{|l|l|}
\hline \multicolumn{1}{|c|}{ Modelo institucional } & \multicolumn{1}{|c|}{ Unidades } \\
\hline Fundação pública & $\begin{array}{l}\text { AL, CE, MA }{ }^{1}, \mathrm{PI}, \mathrm{PE}, \mathrm{RJ}, \mathrm{SP}, \\
\mathrm{RS}, \mathrm{MG}, \mathrm{RO}, \mathrm{MT}, \mathrm{DF}, \mathrm{SC}, \mathrm{BA}^{2}\end{array}$ \\
$\begin{array}{l}\text { Fundo, articulado com Conselho } \\
\text { Estadual de Ciência e Tecnologia }\end{array}$ & $\mathrm{RN}, \mathrm{GO}, \mathrm{MS}, \mathrm{SE}$ \\
$\begin{array}{l}\text { Fundo, articulado com Conselho } \\
\text { Estadual de C\&T e uma fundação }\end{array}$ & $\mathrm{AM}, \mathrm{PB}, \mathrm{PA}$ \\
Apenas Conselho de C\&T & $\mathrm{TO}$ \\
Órgão específico & $\mathrm{PR}^{3}, \mathrm{PA}^{4}$ \\
Não definido & $\mathrm{ES}^{2}$ \\
\hline
\end{tabular}

Notas:

1 Fundação extinta em 1998.

2 As atividades previstas para a FAPBA foram recentemente atribuídas ao CADCT.

3 Em 1998 foi instituído o Fundo Paraná e autorizada a Fundação Araucária e o Paraná Tecnologia.

4 Em 1995 foi instituído o Fundo Estadual de Ciência e Tecnologia com $0.4 \%$ da receita orçamentária.

Até 1998, 22 estados e DF haviam implantado as suas Fundações ou fundos (quadro 2). 
Quadro 2 - Ano de implantação de fundação ou fundo ${ }^{1}$

\begin{tabular}{|l|l|}
\hline Ano & Unidade \\
\hline 1960 & SP \\
1964 & RS \\
1980 & RJ \\
1985 & MG \\
1990 & PE, GO \\
1991 & MS, MA ${ }^{2}, \mathrm{CE}^{3}$ \\
1992 & BA \\
1993 & PB \\
1994 & AL, AC, PI, DF \\
1995 & SE \\
1996 & MT, PA, ES, RN \\
1997 & SC, AP \\
1998 & PR \\
\hline
\end{tabular}

Notas:

1 AM, RO, TO, sem informações.

2 Extinta em 1998.

3 Estatutos só foram aprovados em 1994.

4 Em 1994 foi suprimido o artigo 257 da Constituição do estado passando as atribuições da Fapba ao CADCT.

A referência para cálculo do percentual também varia bastante entre as unidades (quadro 3). 
Quadro 3 - Referência para cálculo do potencial estabelecido

na Constituição estadual

\begin{tabular}{|l|l|}
\hline \multicolumn{1}{|c|}{ Referência } & \multicolumn{1}{|c|}{ Unidade } \\
\hline Receita tributária & AM, BA, GO, MT, RJ, SP, SE, TO, MS, PR \\
Receita orçamentária & DF, ES, PA, PI, PB, PE, RN \\
Receita corrente & MA, MG, SC \\
Receita dos impostos & CE \\
Receita líquida dos impostos & RS \\
Receita estimada & AL \\
Não definida & AC, RO \\
\hline
\end{tabular}

Segundo Ferreira Filho (1993) o total de recursos a serem transferidos por 22 estados e Distrito Federal seria, em 1991, US\$ 317 milhões (US\$ 247 milhões excluindo São Paulo). Em 1992, porém, excluindo São Paulo, as seis fundações e dois fundos já instalados receberam apenas US\$21.3 milhões, correspondentes a $16.9 \%$ do que deveriam receber no período.

Ainda excluindo São Paulo, as demais unidades continuam transferindo os recursos com grande irregularidade, tanto no valor quanto na distribuição ao longo do ano. A tabela 4 reúne seis fundações que vêm atuando com regularidade nos últimos seis anos. Não contando a Fapesp, com a média das transferências anuais de $99 \%$ do devido, nas outras cinco fundações essa média ficou abaixo de 50\%, com a mínima de 14.6\% para Faperj (tabela 4). Nos três anos anteriores (1991, 1992 e 1993) a média foi de 90.7\% para Fapesp, 24.5\% para Fapergs, 8.9\% para Faperj, 25.4\% para Fapemig e $24 \%$ para Fapepe.

Embora os dados reunidos sobre as outras 18 FAPs e fundos não sejam homogêneos quanto a período, eles permitem concluir com razoável aproximação que, de cerca de R \$ 240 milhões devidos por ano, entre 1997 e 1999 foram transferidos cerca de R\$ 60 milhões, dos quais, em alguns casos, uma parcela significativa foi destinada a salários. 
Tabela 4 - Recursos devidos e repassados por cinco estados e DF às suas Fundações de amparo à pesquisa, entre 1994 e 1999, valores em R 1.000

\begin{tabular}{|cl|c|c|c|c|c|c|c}
\hline Estado Recursos & $\mathbf{1 9 9 4}$ & $\mathbf{1 9 9 5}$ & $\mathbf{1 9 9 6}$ & $\mathbf{1 9 9 7}$ & $\mathbf{1 9 9 8}$ & $\mathbf{1 9 9 9}$ & Total \\
\hline \multirow{2}{*}{ SP } & Devido & 77.037 & 141.732 & 181.573 & 184.598 & 190.280 & 204.943 & 983.163 \\
& Transferido & 77.037 & 139.985 & 178.650 & 187.271 & 188.204 & 197.596 & 968.743 \\
& \% do devido & $100 \%$ & $98.7 \%$ & $98.4 \%$ & $101.4 \%$ & $98.9 \%$ & $96.4 \%$ & $99.0 \%^{1}$ \\
& & & & & & & \\
MG $^{2}$ & Devido & 90.049 & 23.405 & 42.500 & 50.838 & 55.587 & 55.567 & 317.945 \\
& Transferido & 5.560 & 13.438 & 35.497 & 28.833 & 33.773 & $16.000^{3}$ & 133.101 \\
& \% do devido & $6.2 \%$ & $57.4 \%$ & $83.6 \%$ & $56.7 \%$ & $60.8 \%$ & $28.8 \%$ & $48.9 \%$ \\
& & & & & & & & \\
$\mathrm{RJ}^{4}$ & Devido & 15.842 & 79.716 & 92.667 & 208.547 & 247.032 & 152.366 & 796.170 \\
& Transferido & 3.402 & 6.223 & 11.245 & 15.168 & 12.026 & 36.415 & 84.479 \\
& \% do devido & $21.5 \%$ & $7.8 \%$ & $12.1 \%$ & $7.3 \%$ & $4.9 \%$ & $23.9 \%$ & $12.9 \%$ \\
& & & & & & & & \\
RS $^{5}$ & Devido & 24.463 & 40.034 & 45.977 & 46.948 & 52.771 & N.D & $210.193^{8}$ \\
& Transferido & 5.121 & 5.071 & 10.500 & 10.717 & 13.644 & N.D & 45.053 \\
& \% do devido & $20.9 \%$ & $12.7 \%$ & $22.8 \%$ & $22.8 \%$ & $25.9 \%$ & - & $21.0 \%$ \\
& & & & & & & & \\
PE $^{6}$ & Devido & 6.872 & 17.000 & 11.349 & 10.948 & 10.728 & $11.000^{9}$ & 66.897 \\
& Transferido & 1.425 & 1.700 & 2.625 & 3.135 & 2.187 & 907 & 11.979 \\
& \% do devido & $20.7 \%$ & $10 \%$ & $23.1 \%$ & $28.6 \%$ & $20.4 \%$ & $8.2 \%$ & $18.5 \%$ \\
& & & & & & & & \\
DF $^{7}$ & Devido & 9.782 & 9.155 & 10.325 & 8.267 & 17.753 & 20.864 & 76.146 \\
& Transferido & 9.528 & 4.158 & 1.921 & 4.692 & 3.777 & 909 & 24.985 \\
& \% do devido & $97.4 \%$ & $45.4 \%$ & $18.6 \%$ & $56.8 \%$ & $21.3 \%$ & $4.3 \%$ & $40.6 \%$ \\
& & & & & & & \\
\hline
\end{tabular}

Notas:

1 Média dos percentuais anuais.

2 Percentual modificado a partir de 1995; ver nota 5 da tabela 3.

3 Até 23 de dezembro de 1999.

4 Além das transferências do estado obteve recursos do governo federal e de rendas próprias, não incluídos no transferido e somando, entre 1994 e 1998, R\$ 32.655.000,00.

5 Como em (4) somando R\$ 15.364.000,00.

6 Como em (4) somando R\$ 7.489.000,00.

7 Como em (4) somando R\$ 6.080,00.

8 Médias para 1995 a 1998.

9 Estimativa, com base nos três anos precedentes.

ND Não disponível 
Nos últimos anos tem havido algumas mudanças de rumo. No Maranhão, a Fapema foi extinta. Na Bahia, mudança na Constituição eliminou a Fapba que estava em discussão, admitindo-se que as suas funções poderiam ser desempenhadas pelo CADCT. A Paraíba, depois de instituir a sua FAP em 1992, extinguiu-a em 1996 e, em 1997, criou a Fapesqpb, com sede em Campina Grande, e a Fapep, com sede em João Pessoa. No Paraná foi instituído o Fundo Paraná, ao qual cabem os $2 \%$ da renda tributária previstos na Constituição, dos quais 30\% no máximo são transferidos à Fundação Araucária cuja estrutura se inspira no modelo das FAPs. Em alguns estados grande parte dos recursos são usados para pagamento de pessoal. O Mato Grosso do Sul reduziu a dotação de $1.5 \%$ para $1 \%$. O percentual de 3\% que vigorava na Fapemig foi reduzido para 0,5\% em 1995, passando a 0,7\% em 1996, 0.8\% em 1997, chegando a 1\% a partir de 1998.

Consideráveis esforços vêm sendo feitos com o objetivo de fortalecer as FAPs, os fundos de amparo à pesquisa e estreitar ligações entre eles e o sistema federal, com o objetivo de fortalecer e descentralizar o sistema nacional de pesquisa e desenvolvimento.

O Fórum Nacional das Fundações, Fundos e Entidades de Amparo à Pesquisa (Forpesq), idealizado em 1996, está elaborando um "Plano Nacional de Fortalecimento das FAPs", no qual se analisa a sua contribuição para "promover o desenvolvimento harmônico de uma política nacional de ciência e tecnologia que contempla a convergência das ações nas três esferas do governo (União, estados e municípios), bem como dos segmentos representativos da sociedade (setores empresarial e político) de modo a garantir que Ciência e Tecnologia, mediante um efetivo Sistema Nacional de Inovação, estejam no epicentro das políticas sustentáveis de desenvolvimento econômico e social do país” (Forpesq, 1999).

É essencial, neste plano, não se perder de vista que a função fundamental das FAPs é contribuir para a formação e fixação de pesquisadores e a criação de condições mínimas locais para desenvolver pesquisa com eficiência e continuidade. Onde tais condições mínimas não forem previamente criadas e progressivamente ampliadas será difícil promover pesquisa em apoio à inovação e ao desenvolvimento.

A Fapesp, que iniciou as suas atividades em 1962, concentrou-se por mais de duas décadas em bolsas para a formação de pesquisadores e auxílios individuais para a pesquisa. Somente nos últimos 15 anos, quando a capacidade científica do estado já estava consideravelmente expandida e diversificada, é que a Fundação introduziu novos modelos de apoio a pesquisa e 
desenvolvimento (8), mas sem prejuízo de auxílios individuais e concessão de bolsas no país e no exterior que, nos últimos cinco anos, somam respectivamente $17.985,24.357$ e 1.843 . Cada um dos novos programas baseia-se em uma rede interdisciplinar de pesquisadores distribuída em diferentes centros para a formação da qual foram básicos os primeiros 20 anos de bolsas e auxílios individuais, com base exclusivamente no mérito científico.

Também outras FAPs, além da concessão de bolsas e auxílios individuais para pesquisa, vêm desenvolvendo novos programas tendo, em muitos casos, o apoio federal por CNPq, Capes e Finep. Em 1991 a Fapergs já havia iniciado um programa em apoio à cooperação universidade-empresa, com 94 projetos aprovados até 1995. A Fapepi lançou o programa piauiense de apoio às tecnologias apropriadas, em conformidade com a realidade regional; a Facepe introduziu em 1995 o seu programa de apoio à capacitação tecnológica das empresas de base local; a Fapesq/PB inclui, entre suas atividades programadas, projetos cooperativos com o setor produtivo primário, secundário e terciário em apoio a projetos de pesquisa e desenvolvimento tecnológico; Faperj, Fapemig, CADCT, entre outros, participam do programa de redes cooperativas (Recope) da Finep. No PADCT está prevista a participação das FAPs, de acordo com as condições em cada caso. Enfim, numerosos convênios com CNPq e Capes abrangem bolsas e várias formas de projetos (Prociência, Nordeste, Softex, Prossiga, Cetem, PhD na Empresa etc.).

Em síntese, o Brasil já conta com instrumentos que lhe permitem descentralizar a política de ciência e tecnologia em apoio à inovação e ao desenvolvimento, atendendo às condições e oportunidades em cada região. As fundações e fundos de amparo à pesquisa são um componente central dessa política desde que se lhes assegurem a autonomia, a regularidade na transferência de recursos (9) e a administração por pesquisadores experientes. A sua função básica é apoiar pesquisa e pós-graduação por meio de bolsas e auxílios para pesquisa e intercâmbio científico em todas as áreas do conhecimento, independentemente da sua aplicação imediata, tendo como base apenas as qualificações do proponente e o mérito e a viabilidade da proposta como condição para formar uma infra-estrutura de pesquisa e recursos humanos suficientemente atualizada e diversificada para dar suporte aos esforços em apoio ao desenvolvimento.

Ao governo federal cumpre formular e desenvolver uma política de ciência e tecnologia que, repetindo as palavras do Forpesq, "contemple as ações nas três esferas de governo (União, estados, municípios) bem como dos segmentos representativos da sociedade (setores empresarial e políti- 
co)". Em grande parte o sucesso dessa política depende de como as fundações e fundos estaduais possam cumprir a parte que lhes compete na promoção do desenvolvimento local. Sem prejuízo da autonomia, a integração com ações e programas do governo federal deverá ser intensificada tanto na execução quanto no financiamento, neste caso quando os recursos locais não forem suficientes para atender às ações prioritárias em apoio ao desenvolvimento científico e tecnológico.

O tempo que se tem para passar de resultados de pesquisa inovação e ganhos em competitividade é cada vez mais curto; a renovação de conhecimentos e prioridades é cada vez mais rápida; a infra-estrutura de apoio em ciências exatas e experimentais tem vida útil cada vez mais curta e custos mais elevados, enquanto a formação de cientistas e centros de pesquisa continua sendo um processo lento, que não dá saltos.

Os países que pretenderem alcançar bons níveis de desenvolvimento econômico e social terão de enfrentar estes desafios com continuidade e com perspectivas de médio e longo prazos. O ritmo de incorporação de resultados de pesquisa em inovação de produtos e serviços pode sofrer flutuações. Mas tanto para responder às demandas espontâneas do sistema produtivo à medida em que ocorrem quanto para encorajar novas demandas, os centros de pesquisa terão de se manter permanentemente ativos, atualizados e diversificados, tanto na formação de conhecimento quanto na formação de novos pesquisadores.

Notas

1 Max Plank Gesellschaft, Fraunhofer Gesellschaft, Helmohltz Zentern, Blauen List e Institutos Federais.

2 Amapá e Roraima passaram à categoria de estado em 1990.

3 Em dezembro de 1948 a Prefeitura de Campo Grande-PB criou a Fundação para o Desenvolvimento da Ciência e da Técnica tendo como dotação o produto da arrecadação do selo municipal de educação e cultura, depositado mensalmente em conta especial no Banco do Nordeste.

4 No caso da FAPESP, até 1985, quando além de se deduzir o transferido aos municípios, as quotas eram transferidas com atraso e desvalorizadas pela inflação, a dotação era, em média, $0,27 \%$ e não $0,5 \%$ como mandava a Constituição de 1947. Atualmente, no cálculo do 1\% previsto na Constituição de 1989, é deduzida a quota de $25 \%$ do ICM transferida aos municípios. 
5 CE, PE, AL, SE, BA, RJ, SP, PR, SC, RS, GO, MT, MS, MG.

6 AC, PI, PE, AL, SP, MT, MG.

7 CE, PA, BA, RJ, SP, MT, PA.

8 Além de alguns "projetos especiais" anteriores (Bioq.-Fapesp, Laboratório de Microeletrônica, Ação Programada em Águas Subterrâneas, Tipologia de Represas, Centro de Bioterismo, Radar Meteorológico, Rede ANSP), a partir de 1985 a Fapesp implantou o programa de projetos temáticos de equipe em apoio à pesquisa interinstitucional e interdisciplinar; recuperação de infra-estrutura para pesquisa em universidades e institutos; formação de centros de pesquisa, inovação e difusão de conhecimento; pesquisa em políticas públicas; inovação tecnológica na pequena empresa; inovação tecnológica em parceria universidade-empresa; capacitação de pessoal técnico em nível médio e superior; apoio à educação (pró-ciências e ensino público); apoio ao jovem pesquisador; biblioteca eletrônica (acesso a revistas do exterior); scielo (divulgação eletrônica da produção científica nacional); estudo do genoma em xylella fastidiosa (clorose variegada da laranja), xanthomonas citri (cancro cítrico), cana-de-açúcar e câncer humano (em colaboração com o Instituto Ludwig de Pesquisa); incentivo ao jornalismo científico; instituto de biodiversidade (Biota-Fapesp).

9 Em alguns casos os percentuais previstos na Constituição podem ser considerados excessivos, resultando em dotações que superam a capacidade do estado de liberá-las ou de utilizá-las adequadamente. Nestes casos, cabe rever a Constituição ajustando os valores à realidade local, como aconteceu em Minas Gerais.

Referências bibliográficas

BUNDSBERICHT FORSCHUNG. Bundsministerium für Bildung, Wissenschaft, Forschung und Technologie. Buchdruckerei, Bonn, Bonner Universitàt, 1996.

FERREIRA FILHO, G.; BAETA NEVES, A. \& CANDROTA, L. F. Fomento à pesquisa e entidades estaduais, FINEP, 1993, 144 p. [mimeo].

FORPESQ. Plano Nacional de Fortalecimento das FAPs. Termos de referência. Recife, FACEPE, 1999 (www.facepe.pe.gov.br/forqpesq).

Alberto Carvalho da Silva, médico, é professor honorário do Instituto de Estudos Avançados da USP. 